\title{
EXISTENCE AND ASYMPTOTIC STABILITY OF SOLUTIONS OF A PERTURBED FRACTIONAL FUNCTIONAL-INTEGRAL EQUATION WITH LINEAR MODIFICATION OF THE ARGUMENT
}

\author{
Mohamed Abdalla Darwish, Johnny Henderson, and Donal O'Regan
}

\begin{abstract}
We study the solvability of a perturbed quadratic functionalintegral equation of fractional order with linear modification of the argument. This equation is considered in the Banach space of real functions defined, bounded and continuous on an unbounded interval. Moreover, we will obtain some asymptotic characterization of solutions.
\end{abstract}

\section{Introduction}

In this paper, we will study the perturbed quadratic fractional functionalintegral equation

$$
x(t)=f(t, x(t))+g\left(t, \frac{h(t, x(t))}{\Gamma(\alpha)} \int_{0}^{t} \frac{u(t, s, x(s), x(\lambda s))}{(t-s)^{1-\alpha}} d s\right),
$$

where $t \in \mathbb{R}_{+}$and $0<\alpha, \lambda<1$. Throughout $f, g, h: \mathbb{R}_{+} \times \mathbb{R} \rightarrow \mathbb{R}$ and $u: \mathbb{R}_{+} \times \mathbb{R}_{+} \times \mathbb{R} \times \mathbb{R} \rightarrow \mathbb{R}$ are functions which satisfy special assumptions (see Section 3). Let us recall that the functions $f=f\left(t, y_{1}\right), g=g\left(t, y_{2}\right)$ and $h=h\left(t, y_{3}\right)$ involved in Eq.(1) generate the superposition operators $F, G$ and $H$, defined by

$$
\begin{aligned}
& \left(F y_{1}\right)(t)=f\left(t, y_{1}(t)\right), \\
& \left(G y_{2}\right)(t)=g\left(t, y_{2}(t)\right),
\end{aligned}
$$

and

$$
\left(H y_{3}\right)(t)=h\left(t, y_{3}(t)\right),
$$

respectively, where $y_{1}=y_{1}(t), y_{2}=y_{2}(t)$ and $y_{3}=y_{3}(t)$ are arbitrary functions defined on $\mathbb{R}_{+}$(see [2]).

We remark that:

Received September 17, 2009.

2010 Mathematics Subject Classification. 45G10, 45M99, 47H08.

Key words and phrases. functional integral equation, fractional integral, perturbed, linear modification of the argument, existence, asymptotic behaviour, measure of noncompactness, Schauder fixed point principle. 
- If $f(t, x)=f(t)$, and $u(t, s, x, y)=u(t, s, x)$ in Eq.(1), then we have a quadratic functional-integral equation of fractional order studied by Darwish and Sadarangani in [18].

- If $f(t, x)=f(t), g(t, v)=v$ and $u(t, s, x, y)=u(t, s, x)$ in Eq.(1), then we have a quadratic Urysohn-Volterra integral equation of fractional order studied by Banaś and O'Regan in [8].

- If $g(t, v)=v$ and $u(t, s, x, y)=u(t, s, x)$ in Eq.(1), then we have a perturbed quadratic integral equation of fractional order studied by Darwish and Henderson in [17].

- If $f(t, x)=a(t)$ and $g(t, v)=v$ in Eq.(1), then we have a quadratic integral equation of fractional order with linear modification of the argument studied by Darwish in [16].

The proofs in $[8,16,18]$ depend on a suitable combination of the technique of measures of noncompactness and the Schauder fixed point principle, while the proof in [17] depends on a suitable combination of the technique of measures of noncompactness and the Darbo fixed point principle.

In the case $\alpha=1, f(t, x)=f(t), g(t, v)=v, h(t, x)=-x$ and $u(t, s, x, y)=$ $k(t, s) x$, Eq.(1) becomes the Volterra counterpart of the following equation

$$
x(t)+x(t) \int_{0}^{t} k(t, s) x(s) d s=f(t), t \in \mathbb{R}_{+} .
$$

Eq.(2) is the nonlinear particle transport equation when removal effects are dominant, where $t$ is the particle speed, the known term $f(t)$ is the intensity of the external source and the unknown function $x(t)$ is related to the particle distribution function $y(t)$ by

$$
x(t)=Q(t) y(t),
$$

where, $Q$ is the positive macroscopic removal collision frequency of the host medium. Finally, the kernel $k(t, s)$ is given by

$$
k(t, s)=\frac{1}{2 t Q(t) Q(s)} \int_{|t-s|}^{t+s} v q(v) d v,
$$

where $q$ is the macroscopic removal collision frequency by the particles between themselves, see $[9,10,13,29]$. On the other hand, Eq.(2) is a generalization of a famous equation in the transport theory, the so-called Chandrasekhar $H$ equation in which $t$ ranges from 0 to $1, f(t)=1, x$ must be identified with the $H$-function, and

$$
k(t, s)=-\frac{t \phi(s)}{t+s}
$$

for a nonnegative characteristic function $\phi$, see $[14,22,25,29]$.

A type of quadratic integral equation arises in the design of bandlimited signals for binary communication using simple memoryless correlation detection, when the signals are disturbed by additive white Gaussian noise. It is shown 
that a bandlimited signal can be designed which eliminates intersymbol interference for signalling at Nyquist rate, this signal is a solution to a quadratic integral equation, see $[1,4,11,14,21,24]$.

Moreover, quadratic integral equations have many other useful applications in describing numerous events and problems of the real world. For example, quadratic integral equations are often applicable in kinetic theory of gases, in the theory of neutron transport, and in the traffic theory, see $[9,10,13,19,21$, $22]$.

The theory of quadratic integral equations with nonsingular kernels has received a lot of attention. Many authors studied the existence of solutions for several classes of nonlinear quadratic integral equations with nonsingular kernels. For example, see Argyros [3], Banaś et al. [6], Caballero et al. [12], Leggett [25], Spiga et al. [29] and Stuart [30], and the references therein.

On the other hand, after the appearance of Darwish's paper [15] there has been significant interest of the study of singular quadratic integral equations or fractional quadratic integral equations, see for example $[8,16,17]$ and the references therein.

The aim of this paper is to prove the existence of solutions of Eq.(1) in the space of real functions, defined, continuous and bounded on an unbounded interval. Moreover, we will obtain some asymptotic characterization of solutions of Eq.(1). Our proof depends on suitable combination of the technique of measures of noncompactness and the Schauder fixed point principle. Finally we note that some of the ideas in this paper are from [8].

\section{Notation and auxiliary facts}

This section is devoted to collecting some definitions and results which will be needed further on. First we recall the definition of the Riemann-Liouville fractional integral, see $[20,23,26,27,28]$ for more information.

Definition 2.1. Let $f \in L_{1}(a, b), 0 \leq a<b<\infty$, and let $\alpha>0$ be a real number. The Riemann-Liouville fractional integral of order $\alpha$ of the function $f(t)$ is defined by

$$
I^{\alpha} f(t)=\frac{1}{\Gamma(\alpha)} \int_{0}^{t} \frac{f(s)}{(t-s)^{1-\alpha}} d s, a<t<b,
$$

where $\Gamma(\alpha)$ denotes the gamma function.

Now, let $(E,\|\cdot\|)$ be an infinite dimensional Banach space with zero element 0 . Let $B(x, r)$ denote the closed ball centered at $x$ with radius $r$. The symbol $B_{r}$ stands for the ball $B(0, r)$.

If $X$ is a subset of $E$, then $\bar{X}$ and $\operatorname{Conv} X$ denote the closure and convex closure of $X$, respectively. Moreover, we denote by $\mathcal{M}_{E}$ the family of all nonempty and bounded subsets of $E$ and by $\mathcal{N}_{E}$ its subfamily consisting of all relatively compact subsets. 
Next we give the definition of the concept of a measure of noncompactness; see [7] and the references therein.

Definition 2.2. A mapping $\mu: \mathcal{M}_{E} \rightarrow \mathbb{R}_{+}=[0, \infty)$ is said to be a measure of noncompactness in $E$ if it satisfies the following conditions:

1) The family $\operatorname{ker} \mu=\left\{X \in \mathcal{M}_{E}: \mu(X)=0\right\}$ is nonempty and $\operatorname{ker} \mu \subset \mathcal{N}_{E}$.

2) $X \subset Y \Rightarrow \mu(X) \leq \mu(Y)$.

3) $\mu(\bar{X})=\mu(\operatorname{Conv} X)=\mu(X)$.

4) $\mu(\lambda X+(1-\lambda) Y) \leq \lambda \mu(X)+(1-\lambda) \mu(Y)$ for $0 \leq \lambda \leq 1$.

5) If $X_{n} \in \mathcal{M}_{E}, X_{n}=\bar{X}_{n}, X_{n+1} \subset X_{n}$ for $n=1,2,3, \ldots$ and $\lim _{n \rightarrow \infty} \mu\left(X_{n}\right)=$ 0 , then $X_{\infty}=\cap_{n=1}^{\infty} X_{n} \neq \phi$.

The family ker $\mu$ described above is called the kernel of the measure of noncompactness $\mu$. Let us observe that the intersection set $X_{\infty}$ from 5 ) belongs to ker $\mu$. In fact, since $\mu\left(X_{\infty}\right) \leq \mu\left(X_{n}\right)$ for every $n$ then we have that $\mu\left(X_{\infty}\right)=0$.

In what follows we will work in the Banach space $B C\left(\mathbb{R}_{+}\right)$consisting of all real functions defined, bounded and continuous on $\mathbb{R}_{+}$. This space is equipped with the standard norm

$$
\|x\|=\sup \{|x(t)|: t \geq 0\} .
$$

Now, we recollect the construction of the measure of noncompactness in $B C\left(\mathbb{R}_{+}\right)$which will be used in the next section (see [7]).

Let us fix a nonempty and bounded subset $X$ of $B C\left(\mathbb{R}_{+}\right)$and numbers $\varepsilon>0$ and $T>0$. For arbitrary function $x \in X$ let us denoted by $\omega^{T}(x, \varepsilon)$ the modulus of continuity of the function $x$ on the interval $[0, T]$, i.e.,

$$
\omega^{T}(x, \varepsilon)=\sup \{|x(t)-x(s)|: t, s \in[0, T],|t-s| \leq \varepsilon\} .
$$

Further, let us put

and

$$
\begin{gathered}
\omega^{T}(X, \varepsilon)=\sup \left\{\omega^{T}(x, \varepsilon): x \in X\right\}, \\
\omega_{0}^{T}(X)=\lim _{\varepsilon \rightarrow 0} \omega^{T}(X, \varepsilon),
\end{gathered}
$$

$$
\omega_{0}^{\infty}(X)=\lim _{T \rightarrow \infty} \omega_{0}^{T}(X) .
$$

Moreover, for a fixed number $t \in \mathbb{R}_{+}$let us define

$$
X(t)=\{x(t): x \in X\}
$$

and

$$
\operatorname{diam} X(t)=\sup \{|x(t)-y(t)|: x, y \in X\} .
$$

Finally, let us define the function $\mu$ on the family $\mathcal{M}_{B C\left(\mathbb{R}_{+}\right)}$by

$$
\mu(X)=\omega_{0}^{\infty}(X)+c(X),
$$

where $c(X)=\limsup \operatorname{diam} X(t)$. The function $\mu$ is a measure of noncompactness in the space $B C\left(\mathbb{R}_{+}\right)$, see [5]. 
Let us mention that the kernel ker $\mu$ of the measure $\mu$ consists of all sets $X \in \mathcal{M}_{B C\left(\mathbb{R}_{+}\right)}$such that functions from $X$ are locally equicontinuous on $\mathbb{R}_{+}$ and the thickness of the bundle formed by functions belonging to the set $X$ tends to zero at infinity.

For further purposes we recall the definition of the concept of the asymptotic stability which will be used in our considerations.

To this end assume that $\Omega$ is a nonempty subset of the space $B C\left(\mathbb{R}_{+}\right)$. Let $Q$ : $\Omega \rightarrow B C\left(\mathbb{R}_{+}\right)$be a given operator. Consider the following operator equation

$$
x(t)=(Q x)(t), \quad t \in \mathbb{R}_{+} .
$$

Definition 2.3. We say that solutions of the above equation are asymptotically stable if there exists a ball $B\left(x_{0}, r\right)$ such that $\Omega \cap B\left(x_{0}, r\right) \neq \emptyset$ and such that for each $\varepsilon>0$ there exists $T>0$ such that for arbitrary solutions $x=x(t), y=$ $y(t)$ of this equation belonging to $\Omega \cap B\left(x_{0}, r\right)$ the inequality $|x(t)-y(t)| \leq \varepsilon$ is satisfied for any $t \geq T$.

\section{Main theorem}

In this section we will study Eq.(1) assuming that the following are satisfied:

$\left(a_{1}\right) f, g: \mathbb{R}_{+} \times \mathbb{R} \rightarrow \mathbb{R}$ are continuous and the functions $t \rightarrow f(t, 0)$ and $t \rightarrow g(t, 0)$ are bounded on $\mathbb{R}_{+}$with $f^{*}=\sup \left\{|f(t, 0)|: t \in \mathbb{R}_{+}\right\}$and $g^{*}=\sup \left\{|g(t, 0)|: t \in \mathbb{R}_{+}\right\}$. Moreover, there exist continuous functions $l(t)=l: \mathbb{R}_{+} \rightarrow \mathbb{R}_{+}$and $m(t)=m: \mathbb{R}_{+} \rightarrow \mathbb{R}_{+}$such that

$$
|f(t, x)-f(t, y)| \leq l(t)|x-y|
$$

and

$$
|g(t, x)-g(t, y)| \leq m(t)|x-y|
$$

for all $x, y \in \mathbb{R}$ and for any $t \in \mathbb{R}_{+}$.

$\left(a_{2}\right) h: \mathbb{R}_{+} \times \mathbb{R} \rightarrow \mathbb{R}$ is continuous and there exists a continuous function $n(t)=n: \mathbb{R}_{+} \rightarrow \mathbb{R}_{+}$such that

$$
|h(t, x)-h(t, y)| \leq n(t)|x-y|
$$

for all $x, y \in \mathbb{R}$ and for any $t \in \mathbb{R}_{+}$.

$\left(a_{3}\right) u: \mathbb{R}_{+} \times \mathbb{R}_{+} \times \mathbb{R} \times \mathbb{R} \rightarrow \mathbb{R}$ is a continuous function. Moreover, there exist a function $q(t)=q: \mathbb{R}_{+} \rightarrow \mathbb{R}_{+}$being continuous on $\mathbb{R}_{+}$and a function $\Phi: \mathbb{R}_{+} \times \mathbb{R}_{+} \rightarrow \mathbb{R}_{+}$being continuous and nondecreasing on $\mathbb{R}_{+}$with $\Phi(0,0)=0$ and such that

$$
\left|u\left(t, s, x_{2}, y_{2}\right)-u\left(t, s, x_{1}, y_{1}\right)\right| \leq q(t) \Phi\left(\left|x_{2}-x_{1}\right|,\left|y_{2}-y_{1}\right|\right)
$$

for all $t, s \in \mathbb{R}_{+}$such that $t \geq s$ and for all $x_{i}, y_{i} \in \mathbb{R}(i=1,2)$.

For further purposes let us define the function $u^{*}: \mathbb{R}_{+} \rightarrow \mathbb{R}_{+}$by

$$
u^{*}(t)=\max \{|u(t, s, 0,0)|: 0 \leq s \leq t\} .
$$


$\left(a_{4}\right)$ The functions $\phi, \psi, \xi, \eta: \mathbb{R}_{+} \rightarrow \mathbb{R}_{+}$defined by

$$
\begin{aligned}
\phi(t) & =m(t) n(t) q(t) t^{\alpha}, \\
\psi(t) & =m(t) n(t) u^{*}(t) t^{\alpha}, \\
\xi(t) & =m(t) q(t)|h(t, 0)| t^{\alpha}
\end{aligned}
$$

and $\eta(t)=m(t) u^{*}(t)|h(t, 0)| t^{\alpha}$ are bounded on $\mathbb{R}_{+}$and the functions $\phi$ and $\xi$ vanish at infinity, i.e., $\lim _{t \rightarrow \infty} \phi(t)=\lim _{t \rightarrow \infty} \xi(t)=0$.

$\left(a_{5}\right)$ There exists a positive solution $r_{0}$ of the inequality

(4) $\left(l^{*} r+f^{*}+g^{*}\right) \Gamma(\alpha+1)+\left[\phi^{*} r \Phi(r, r)+\psi^{*} r+\xi^{*} \Phi(r, r)+\eta^{*}\right] \leq r \Gamma(\alpha+1)$ and $l^{*} \Gamma(\alpha+1)+\phi^{*} \Phi\left(r_{0}, r_{0}\right)+\psi^{*}<\Gamma(\alpha+1)$, where $l^{*}=\sup \{l(t):$ $\left.t \in \mathbb{R}_{+}\right\}, \phi^{*}=\sup \left\{\phi(t): t \in \mathbb{R}_{+}\right\}, \psi^{*}=\sup \left\{\psi(t): t \in \mathbb{R}_{+}\right\}, \xi^{*}=$ $\sup \left\{\xi(t): t \in \mathbb{R}_{+}\right\}$and $\eta^{*}=\sup \left\{\eta(t): t \in \mathbb{R}_{+}\right\}$.

Now, we are in a position to state and prove our main result.

Theorem 3.1. Let the assumptions $\left(a_{1}\right)-\left(a_{5}\right)$ be satisfied. Then Eq.(1) has at least one solution $x \in B C\left(\mathbb{R}_{+}\right)$and all solutions of this equation belonging to the ball $B_{r_{0}}$ are asymptotically stable.

Proof. Denote by $\mathcal{F}$ the operator associated with the right-hand side of Eq.(1), i.e., Eq.(1) takes the form

$$
x=\mathcal{F} x,
$$

where

$$
\begin{gathered}
\mathcal{F} x=F x+G \mathcal{H} x, \\
(\mathcal{H} x)(t)=(H x)(t) \cdot(\mathcal{U} x)(t)
\end{gathered}
$$

and

$$
(\mathcal{U} x)(t)=\frac{1}{\Gamma(\alpha)} \int_{0}^{t} \frac{u(t, s, x(s), x(\lambda s))}{(t-s)^{1-\alpha}} d s, t \in \mathbb{R}_{+} .
$$

Solving Eq.(1) is equivalent to finding a fixed point of the operator $\mathcal{F}$ defined on the space $B C\left(\mathbb{R}_{+}\right)$.

We claim that for any function $x \in B C\left(\mathbb{R}_{+}\right)$the operator $\mathcal{F}$ is continuous on $\mathbb{R}_{+}$. To establish this claim it suffices to show that if $x \in B C\left(\mathbb{R}_{+}\right)$, then $\mathcal{U} x$ is continuous function on $\mathbb{R}_{+}$, thanks to $\left(a_{1}\right),\left(a_{2}\right)$ and $\left(a_{3}\right)$. For, take an arbitrary $x \in B C\left(\mathbb{R}_{+}\right)$and fix $\varepsilon>0$ and $T>0$. Assume that $t_{1}, t_{2} \in \mathbb{R}_{+}$are such that $\left|t_{2}-t_{1}\right| \leq \varepsilon$. Without loss of generality we can assume that $t_{2}>t_{1}$. Then we get

$$
\begin{aligned}
& \left|(\mathcal{U} x)\left(t_{2}\right)-(\mathcal{U} x)\left(t_{1}\right)\right| \\
= & \left|\frac{1}{\Gamma(\alpha)} \int_{0}^{t_{2}} \frac{u\left(t_{2}, s, x(s), x(\lambda s)\right)}{\left(t_{2}-s\right)^{1-\alpha}} d s-\frac{1}{\Gamma(\alpha)} \int_{0}^{t_{1}} \frac{u\left(t_{1}, s, x(s), x(\lambda s)\right)}{\left(t_{1}-s\right)^{1-\alpha}} d s\right| \\
\leq & \left|\frac{1}{\Gamma(\alpha)} \int_{0}^{t_{2}} \frac{u\left(t_{2}, s, x(s), x(\lambda s)\right)}{\left(t_{2}-s\right)^{1-\alpha}} d s-\frac{1}{\Gamma(\alpha)} \int_{0}^{t_{1}} \frac{u\left(t_{2}, s, x(s), x(\lambda s)\right)}{\left(t_{2}-s\right)^{1-\alpha}} d s\right|
\end{aligned}
$$




$$
\begin{aligned}
& +\left|\frac{1}{\Gamma(\alpha)} \int_{0}^{t_{1}} \frac{u\left(t_{2}, s, x(s), x(\lambda s)\right)}{\left(t_{2}-s\right)^{1-\alpha}} d s-\frac{1}{\Gamma(\alpha)} \int_{0}^{t_{1}} \frac{u\left(t_{1}, s, x(s), x(\lambda s)\right)}{\left(t_{2}-s\right)^{1-\alpha}} d s\right| \\
& +\left|\frac{1}{\Gamma(\alpha)} \int_{0}^{t_{1}} \frac{u\left(t_{1}, s, x(s), x(\lambda s)\right)}{\left(t_{2}-s\right)^{1-\alpha}} d s-\frac{1}{\Gamma(\alpha)} \int_{0}^{t_{1}} \frac{u\left(t_{1}, s, x(s), x(\lambda s)\right)}{\left(t_{1}-s\right)^{1-\alpha}} d s\right| \\
\leq & \frac{1}{\Gamma(\alpha)} \int_{t_{1}}^{t_{2}} \frac{\left|u\left(t_{2}, s, x(s), x(\lambda s)\right)\right|}{\left(t_{2}-s\right)^{1-\alpha}} d s \\
& +\frac{1}{\Gamma(\alpha)} \int_{0}^{t_{1}} \frac{\left|u\left(t_{2}, s, x(s), x(\lambda s)\right)-u\left(t_{1}, s, x(s), x(\lambda s)\right)\right|}{\left(t_{2}-s\right)^{1-\alpha}} d s \\
& +\frac{1}{\Gamma(\alpha)} \int_{0}^{t_{1}}\left|u\left(t_{1}, s, x(s), x(\lambda s)\right)\right|\left[\left(t_{1}-s\right)^{\alpha-1}-\left(t_{2}-s\right)^{\alpha-1}\right] d s .
\end{aligned}
$$

Therefore, if we denote

$$
\begin{array}{r}
\omega_{d}^{T}(u, \varepsilon)=\sup \left\{\left|u\left(t_{2}, s, y, \hat{y}\right)-u\left(t_{1}, s, y, \hat{y}\right)\right|: s, t_{1}, t_{2} \in[0, T],\right. \\
\left.t_{1} \geq s, t_{2} \geq s,\left|t_{2}-t_{1}\right| \leq \varepsilon, \text { and } y, \hat{y} \in[-d, d]\right\},
\end{array}
$$

then we obtain

$$
\begin{aligned}
& \left|(\mathcal{U} x)\left(t_{2}\right)-(\mathcal{U} x)\left(t_{1}\right)\right| \\
\leq & \frac{1}{\Gamma(\alpha)} \int_{t_{1}}^{t_{2}} \frac{\left|u\left(t_{2}, s, x(s), x(\lambda s)\right)-u\left(t_{2}, s, 0,0\right)\right|+\left|u\left(t_{2}, s, 0,0\right)\right|}{\left(t_{2}-s\right)^{1-\alpha}} d s \\
& +\frac{1}{\Gamma(\alpha)} \int_{0}^{t_{1}} \frac{\omega_{\|x\|}^{T}(u, \varepsilon)}{\left(t_{2}-s\right)^{1-\alpha}} d s \\
& +\frac{1}{\Gamma(\alpha)} \int_{0}^{t_{1}}\left[\left|u\left(t_{1}, s, x(s), x(\lambda s)\right)-u\left(t_{1}, s, 0,0\right)\right|+\left|u\left(t_{1}, s, 0,0\right)\right|\right] \\
\leq & \frac{1}{\Gamma(\alpha)} \int_{t_{1}}^{t_{2}} \frac{q\left(t_{2}\right) \Phi(|x(s)|,|x(\lambda s)|)+u^{*}\left(t_{2}\right)}{\left(t_{2}-s\right)^{1-\alpha}} d s \\
& +\frac{\omega_{\|x\|}^{T}}{\Gamma(\alpha+1)}\left[t_{2}\left(t_{2}^{\alpha}-\left(t_{2}-t_{1}\right)^{\alpha}\right]\right. \\
& +\frac{1}{\Gamma(\alpha)} \int_{0}^{t_{1}}\left[q\left(t_{1}\right) \Phi(|x(s)|,|x(\lambda s)|)+u^{*}\left(t_{1}\right)\right]\left[\left(t_{1}-s\right)^{\alpha-1}-\left(t_{2}-s\right)^{\alpha-1}\right] d s \\
\leq & \frac{q\left(t_{2}\right) \Phi(\|x\|,\|x\|)+u^{*}\left(t_{2}\right)}{\Gamma(\alpha+1)}\left(t_{2}-t_{1}\right)^{\alpha}+\frac{\omega_{\|x\|}^{T}(u, \varepsilon)}{\Gamma(\alpha+1)} t_{2}^{\alpha} \\
& +\frac{q\left(t_{1}\right) \Phi(\|x\|,\|x\|)+u^{*}\left(t_{1}\right)}{\Gamma(\alpha+1)}\left[t_{1}^{\alpha}-t_{2}^{\alpha}+\left(t_{2}-t_{1}\right)^{\alpha}\right] .
\end{aligned}
$$

Thus

(8) $\omega^{T}(\mathcal{U} x, \varepsilon) \leq \frac{1}{\Gamma(\alpha+1)}\left\{2 \varepsilon^{\alpha}[\hat{q}(T) \Phi(\|x\|,\|x\|)+\hat{u}(T)]+T^{\alpha} \omega_{\|x\|}^{T}(u, \varepsilon)\right\}$, 
where

and

$$
\hat{q}(T)=\max \{q(t): t \in[0, T]\}
$$

$$
\hat{u}(T)=\max \left\{u^{*}(t): t \in[0, T]\right\} .
$$

In view of the uniform continuity of the function $u$ on $[0, T] \times[0, T] \times$ $[-\|x\|,\|x\|] \times[-\|x\|,\|x\|]$ we have that $\omega_{\|x\|}^{T}(u, \varepsilon) \rightarrow 0$ as $\varepsilon \rightarrow 0$. From the above inequality we infer that the function $\mathcal{U} x$ is continuous on the interval $[0, T]$ for any $T>0$. This yields the continuity of $\mathcal{U} x$ on $\mathbb{R}_{+}$and, consequently, the function $\mathcal{F} x$ is continuous on $\mathbb{R}_{+}$.

Now, we show that $\mathcal{F} x$ is bounded on $\mathbb{R}_{+}$. Indeed, in view of our hypotheses for arbitrary $x \in B C\left(\mathbb{R}_{+}\right)$and for a fixed $t \in \mathbb{R}_{+}$we have

$$
\begin{aligned}
& |(\mathcal{F} x)(t)| \\
\leq & \left|f(t, x(t))+g\left(t, \frac{h(t, x(t))}{\Gamma(\alpha)} \int_{0}^{t} \frac{u(t, s, x(s), x(\lambda s))}{(t-s)^{1-\alpha}} d s\right)\right| \\
\leq & l(t)\|x\|+|f(t, 0)|+\frac{m(t)}{\Gamma(\alpha)}[|h(t, x(t))-h(t, 0)|+|h(t, 0)|] \\
& \quad \times \int_{0}^{t} \frac{|u(t, s, x(s), x(\lambda s))-u(t, s, 0,0)|+|u(t, s, 0,0)|}{(t-s)^{1-\alpha}} d s+|g(t, 0)| \\
\leq & l^{*}\|x\|+f^{*}+g^{*}+\frac{m(t)[n(t)\|x\|+|h(t, 0)|]}{\Gamma(\alpha)} \int_{0}^{t} \frac{q(t) \Phi(|x(s)|,|x(\lambda s)|)+u^{*}(t)}{(t-s)^{1-\alpha}} d s \\
\leq & l^{*}\|x\|+f^{*}+g^{*}+\frac{m(t)[n(t)\|x\|+|h(t, 0)|]}{\Gamma(\alpha+1)}\left[q(t) \Phi(\|x\|,\|x\|)+u^{*}(t)\right] t^{\alpha} \\
= & l^{*}\|x\|+f^{*}+g^{*}+\frac{1}{\Gamma(\alpha+1)}[\phi(t)\|x\| \Phi(\|x\|,\|x\|)+\psi(t)\|x\| \\
& +\xi(t) \Phi(\|x\|,\|x\|)+\eta(t)] .
\end{aligned}
$$

Hence, $\mathcal{F} x$ is bounded on $\mathbb{R}_{+}$, thanks to assumption $\left(a_{4}\right)$. This assertion in conjunction with the continuity of $\mathcal{F} x$ on $\mathbb{R}_{+}$allows us to conclude that the operator $\mathcal{F}$ maps $B C\left(\mathbb{R}_{+}\right)$into itself. Moreover, from the last estimate we have $\|\mathcal{F} x\| \leq l^{*}\|x\|+f^{*}+g^{*}+\frac{1}{\Gamma(\alpha+1)}\left[\phi^{*}\|x\| \Phi(\|x\|,\|x\|)+\psi^{*}\|x\|+\xi^{*} \Phi(\|x\|,\|x\|)+\eta^{*}\right]$.

Linking this estimate with assumption $\left(a_{5}\right)$ we deduce that there exists $r_{0}>0$ such that the operator $\mathcal{F}$ transforms the ball $B_{r_{0}}$ into itself.

In what follows let us take a nonempty set $X \subset B_{r_{0}}$. Then, for arbitrary $x, y \in X$ and for a fixed $t \in \mathbb{R}_{+}$, we obtain

$$
\begin{aligned}
& |(\mathcal{F} x)(t)-(\mathcal{F} y)(t)| \\
\leq l(t)|x(t)-y(t)|+\frac{m(t)}{\Gamma(\alpha)} \mid & h(t, x(t)) \int_{0}^{t} \frac{u(t, s, x(s), x(\lambda s))}{(t-s)^{1-\alpha}} d s \\
& \quad-h(t, y(t)) \int_{0}^{t} \frac{u(t, s, y(s), y(\lambda s))}{(t-s)^{1-\alpha}} d s \mid
\end{aligned}
$$




$$
\begin{aligned}
& \leq l(t)|x(t)-y(t)|+\frac{m(t)|h(t, x(t))-h(t, y(t))|}{\Gamma(\alpha)} \int_{0}^{t} \frac{|u(t, s, x(s), x(\lambda s))|}{(t-s)^{1-\alpha}} d s \\
& +\frac{m(t)|h(t, y(t))|}{\Gamma(\alpha)} \int_{0}^{t} \frac{|u(t, s, x(s), x(\lambda s))-u(t, s, y(s), y(\lambda s))|}{(t-s)^{1-\alpha}} d s \\
& \leq l(t)|x(t)-y(t)|+\frac{m(t) n(t)|x(t)-y(t)|}{\Gamma(\alpha)} \\
& \times \int_{0}^{t} \frac{|u(t, s, x(s), x(\lambda s))-u(t, s, 0,0)|+|u(t, s, 0,0)|}{(t-s)^{1-\alpha}} d s \\
& +\frac{m(t)[n(t)|y(t)|+|h(t, 0)|]}{\Gamma(\alpha)} \int_{0}^{t} \frac{q(t) \Phi(|x(s)-y(s)|,|x(\lambda s)-y(\lambda s)|)}{(t-s)^{1-\alpha}} d s \\
& \leq l(t)|x(t)-y(t)|+\frac{m(t) n(t)|x(t)-y(t)|}{\Gamma(\alpha)} \int_{0}^{t} \frac{q(t) \Phi(|x(s)|,|x(\lambda s)|)+u^{*}(t)}{(t-s)^{1-\alpha}} d s \\
& +\frac{m(t)[n(t)|y(t)|+|h(t, 0)|]}{\Gamma(\alpha)} \int_{0}^{t} \frac{q(t) \Phi(|x(s)|+|y(s)|,|x(\lambda t)|+|y(\lambda t)|)}{(t-s)^{1-\alpha}} d s \\
& \leq l(t)|x(t)-y(t)|+\frac{m(t) n(t) q(t)(|x(t)|+|y(t)|)}{\Gamma(\alpha)} \int_{0}^{t} \frac{\Phi(|x(s)|,|x(\lambda s)|)}{(t-s)^{1-\alpha}} d s \\
& +\frac{m(t) n(t) u^{*}(t)|x(t)-y(t)|}{\Gamma(\alpha)} \int_{0}^{t} \frac{d s}{(t-s)^{1-\alpha}} \\
& +\frac{m(t) n(t) q(t)|y(t)|}{\Gamma(\alpha)} \int_{0}^{t} \frac{\Phi(|x(s)|+|y(s)|,|x(\lambda t)|+|y(\lambda t)|)}{(t-s)^{1-\alpha}} d s \\
& +\frac{m(t) q(t)|h(t, 0)|}{\Gamma(\alpha)} \int_{0}^{t} \frac{\Phi(|x(s)|+|y(s)|,|x(\lambda t)|+|y(\lambda t)|)}{(t-s)^{1-\alpha}} d s \\
& \leq l(t) \operatorname{diam} X(t)+\frac{2 m(t) n(t) q(t) r_{0} \Phi\left(r_{0}, r_{0}\right)}{\Gamma(\alpha)} \int_{0}^{t} \frac{d s}{(t-s)^{1-\alpha}} \\
& +\frac{m(t) n(t) u^{*}(t) \operatorname{diam} X(t)}{\Gamma(\alpha)} \int_{0}^{t} \frac{d s}{(t-s)^{1-\alpha}} \\
& +\frac{m(t) n(t) q(t) r_{0} \Phi\left(2 r_{0}, 2 r_{0}\right)}{\Gamma(\alpha)} \int_{0}^{t} \frac{d s}{(t-s)^{1-\alpha}} \\
& +\frac{m(t) q(t)|h(t, 0)| \Phi\left(2 r_{0}, 2 r_{0}\right)}{\Gamma(\alpha)} \int_{0}^{t} \frac{d s}{(t-s)^{1-\alpha}} \\
& \leq l(t) \operatorname{diam} X(t)+\frac{2 \phi(t) r_{0} \Phi\left(r_{0}, r_{0}\right)}{\Gamma(\alpha+1)}+\frac{\psi(t)}{\Gamma(\alpha+1)} \operatorname{diam} X(t)+\frac{\phi(t) r_{0} \Phi\left(2 r_{0}, 2 r_{0}\right)}{\Gamma(\alpha+1)} \\
& +\frac{\xi(t) \Phi\left(2 r_{0}, 2 r_{0}\right)}{\Gamma(\alpha+1)}
\end{aligned}
$$

Hence, we can easily deduce the following inequality

$$
\operatorname{diam}(\mathcal{F} X)(t) \leq l(t) \operatorname{diam} X(t)+\frac{2 \phi(t) r_{0} \Phi\left(r_{0}, r_{0}\right)}{\Gamma(\alpha+1)}+\frac{\psi(t)}{\Gamma(\alpha+1)} \operatorname{diam} X(t)
$$




$$
+\frac{\phi(t) r_{0} \Phi\left(2 r_{0}, 2 r_{0}\right)}{\Gamma(\alpha+1)}+\frac{\xi(t) \Phi\left(2 r_{0}, 2 r_{0}\right)}{\Gamma(\alpha+1)} .
$$

Now, taking into account hypothesis $\left(a_{5}\right)$ we obtain

$$
c(\mathcal{F} X) \leq k c(X),
$$

where $k=l^{*}+\frac{\phi^{*} \Phi\left(r_{0}, r_{0}\right)+\psi^{*}}{\Gamma(\alpha+1)} \geq l^{*}+\frac{\psi^{*}}{\Gamma(\alpha+1)}$. Obviously, in view of hypothesis $\left(a_{5}\right)$ we have that $k<1$.

In what follows, let us take arbitrary numbers $\varepsilon>0$ and $T>0$. Choose a function $x \in X$ and take $t_{1}, t_{2} \in[0, T]$ such that $\left|t_{2}-t_{1}\right| \leq \varepsilon$. Without loss of generality we can assume that $t_{2}>t_{1}$. Then, taking into account our hypotheses and (8), we have

$$
\begin{aligned}
& \left|(\mathcal{F} x)\left(t_{2}\right)-(\mathcal{F} x)\left(t_{1}\right)\right| \\
\leq & \left|(F x)\left(t_{2}\right)-(F x)\left(t_{1}\right)\right|+m\left(t_{2}\right)\left|(H x)\left(t_{2}\right)(\mathcal{U} x)\left(t_{2}\right)-(H x)\left(t_{1}\right)(\mathcal{U} x)\left(t_{2}\right)\right| \\
& +m\left(t_{2}\right)\left|(H x)\left(t_{1}\right)(\mathcal{U} x)\left(t_{2}\right)-(H x)\left(t_{1}\right)(\mathcal{U} x)\left(t_{1}\right)\right| \\
& +\left|g\left(t_{2},(H x)\left(t_{1}\right)(\mathcal{U} x)\left(t_{1}\right)\right)-g\left(t_{1},(H x)\left(t_{1}\right)(\mathcal{U} x)\left(t_{1}\right)\right)\right| \\
\leq & l(t)\left|x\left(t_{2}\right)-x\left(t_{1}\right)\right|+\omega_{f}^{T}(\varepsilon)+\frac{m\left(t_{2}\right)\left|h\left(t_{2}, x\left(t_{2}\right)\right)-h\left(t_{1}, x\left(t_{1}\right)\right)\right|}{\Gamma(\alpha)} \\
& \quad \times \int_{0}^{t_{2}} \frac{\left|u\left(t_{2}, s, x(s), x(\lambda s)\right)-u\left(t_{2}, s, 0,0\right)\right|+\left|u\left(t_{2}, s, 0,0\right)\right|}{\left.t_{2}-s\right)^{1-\alpha}} d s \\
& +\frac{m\left(t_{2}\right)\left[\left|h\left(t_{1}, x\left(t_{1}\right)\right)-h\left(t_{1}, 0\right)\right|+\left|h\left(t_{1}, 0\right)\right|\right]}{\Gamma(\alpha+1)} \\
& \quad \times\left\{2 \varepsilon^{\alpha}[\hat{q}(T) \Phi(\|x\|,\|x\|)+\hat{u}(T)]+T^{\alpha} \omega_{\|x\|}^{T}(u, \varepsilon)\right\} \\
& +\left|g\left(t_{2},(H x)\left(t_{1}\right)(\mathcal{U} x)\left(t_{1}\right)\right)-g\left(t_{1},(H x)\left(t_{1}\right)(\mathcal{U} x)\left(t_{1}\right)\right)\right| \\
\leq & l(t) \omega^{T}(x, \varepsilon)+\omega_{f}^{T}(\varepsilon)+\frac{m\left(t_{2}\right)\left[n\left(t_{2}\right)\left|x\left(t_{2}\right)-x\left(t_{1}\right)\right|+\omega_{h}^{T}(\varepsilon)\right]}{\Gamma(\alpha)} \\
& \quad \times \int_{0}^{t_{2}} \frac{q\left(t_{2}\right) \Phi(|x(s)|,|x(\lambda s)|)+u^{*}\left(t_{2}\right)}{\left(t_{2}-s\right)^{1-\alpha}} d s \\
& +\frac{m\left(t_{2}\right)\left[n\left(t_{1}\right)\left|x\left(t_{1}\right)\right|+\left|h\left(t_{1}, 0\right)\right|\right]}{\Gamma(\alpha+1)}\left\{2 \varepsilon^{\alpha}[\hat{l}(T) \Phi(\|x\|,\|x\|)+\hat{u}(T)]+T^{\alpha} \omega_{\|x\|}^{T}(u, \varepsilon)\right\} \\
& +\left|g\left(t_{2},(H x)\left(t_{1}\right)(\mathcal{U} x)\left(t_{1}\right)\right)-g\left(t_{1},(H x)\left(t_{1}\right)(\mathcal{U} x)\left(t_{1}\right)\right)\right| \\
\leq & l(t) \omega^{T}(x, \varepsilon)+\omega_{f}^{T}(\varepsilon)+\frac{t_{2}^{\alpha}}{\Gamma(\alpha+1)} m\left(t_{2}\right)\left[n\left(t_{2}\right) \omega^{T}(x, \varepsilon)+\omega_{h}^{T}(\varepsilon)\right]\left[q\left(t_{2}\right) \Phi\left(r_{0}\right)+u^{*}\left(t_{2}\right)\right] \\
& +\frac{\hat{m}(T)\left[n\left(t_{1}\right) r_{0}+\hat{h}(T)\right]}{\Gamma(\alpha+1)}\left\{2 \varepsilon^{\alpha}[\hat{q}(T) \Phi(\|x\|,\|x\|)+\hat{u}(T)]+T^{\alpha} \omega_{\|x\|}^{T}(u, \varepsilon)\right\} \\
& +\left|g\left(t_{2},(H x)\left(t_{1}\right)(\mathcal{U} x)\left(t_{1}\right)\right)-g\left(t_{1},(H x)\left(t_{1}\right)(\mathcal{U} x)\left(t_{1}\right)\right)\right| \\
\leq & \omega_{f}^{T}(\varepsilon)+\frac{\left[\Gamma(\alpha+1) l\left(t_{2}\right)+\phi\left(t_{2}\right) \Phi\left(r_{0}, r_{0}\right)+\psi\left(t_{2}\right)\right]}{\Gamma(\alpha+1)} \omega^{T}(x, \varepsilon) \\
& +\frac{T^{\alpha} \omega_{h}^{T}(\varepsilon)}{\Gamma(\alpha+1)} \hat{m}(T)\left[\hat{q}(T) \Phi\left(r_{0}, r_{0}\right)+\hat{u}(T)\right]
\end{aligned}
$$




$$
\begin{aligned}
& +\frac{\hat{m}(T)\left[\hat{n}(T) r_{0}+\hat{h}(T)\right]}{\Gamma(\alpha+1)}\left\{2 \varepsilon^{\alpha}[\hat{q}(T) \Phi(\|x\|,\|x\|)+\hat{u}(T)]+T^{\alpha} \omega_{\|x\|}^{T}(u, \varepsilon)\right\} \\
& +\left|g\left(t_{2},(H x)\left(t_{1}\right)(\mathcal{U} x)\left(t_{1}\right)\right)-g\left(t_{1},(H x)\left(t_{1}\right)(\mathcal{U} x)\left(t_{1}\right)\right)\right| \\
\leq & \omega_{f}^{T}(\varepsilon)+\left[l^{*}+\frac{\phi^{*} \Phi\left(r_{0}, r_{0}\right)+\psi^{*}}{\Gamma(\alpha+1)}\right] \omega^{T}(x, \varepsilon)+\frac{T^{\alpha} \omega_{h}^{T}(\varepsilon)}{\Gamma(\alpha+1)} \hat{m}(T)\left[\hat{q}(T) \Phi\left(r_{0}, r_{0}\right)+\hat{u}(T)\right] \\
& +\frac{\hat{m}(T)\left[\hat{n}(T) r_{0}+\hat{h}(T)\right]}{\Gamma(\alpha+1)}\left\{2 \varepsilon^{\alpha}[\hat{q}(T) \Phi(\|x\|,\|x\|)+\hat{u}(T)]+T^{\alpha} \omega_{\|x\|}^{T}(u, \varepsilon)\right\} \\
& +\left|g\left(t_{2},(H x)\left(t_{1}\right)(\mathcal{U} x)\left(t_{1}\right)\right)-g\left(t_{1},(H x)\left(t_{1}\right)(\mathcal{U} x)\left(t_{1}\right)\right)\right|,
\end{aligned}
$$

where

$$
\begin{aligned}
\omega_{\mu}^{T}(\varepsilon)=\sup \left\{\left|\mu\left(t_{2}, x\right)-\mu\left(t_{1}, x\right)\right|: t_{1}, t_{2} \in[0, T],\left|t_{2}-t_{1}\right| \leq \varepsilon, x \in\left[-r_{0}, r_{0}\right]\right\}, \\
\hat{n}(T)=\max \{n(t): t \in[0, T]\}, \\
\hat{m}(T)=\max \{m(t): t \in[0, T]\}, \\
\hat{q}(T)=\max \{q(t): t \in[0, T]\},
\end{aligned}
$$

and

Hence,

$$
\hat{h}(T)=\max \{|h(t, 0)|: t \in[0, T]\}
$$

$$
\begin{aligned}
& \omega^{T}(\mathcal{F} x, \varepsilon) \\
\leq & \omega_{f}^{T}(\varepsilon)+\left[l^{*}+\frac{\phi^{*} \Phi\left(r_{0}, r_{0}\right)+\psi^{*}}{\Gamma(\alpha+1)}\right] \omega^{T}(x, \varepsilon)+\frac{T^{\alpha} \omega_{h}^{T}(\varepsilon)}{\Gamma(\alpha+1)} \hat{m}(T)\left[\hat{l}(T) \Phi\left(r_{0}\right)+\hat{u}(T)\right] \\
& +\frac{\hat{m}(T)\left[\hat{n}(T) r_{0}+\hat{h}(T)\right]}{\Gamma(\alpha+1)}\left\{2 \varepsilon^{\alpha}[\hat{q}(T) \Phi(\|x\|,\|x\|)+\hat{u}(T)]+T^{\alpha} \omega_{\|x\|}^{T}(u, \varepsilon)\right\} \\
& +\sup \left\{\left|g\left(t_{2},(H x)\left(t_{1}\right)(\mathcal{U} x)\left(t_{1}\right)\right)-g\left(t_{1},(H x)\left(t_{1}\right)(\mathcal{U} x)\left(t_{1}\right)\right)\right|: t_{1}, t_{2} \in[0, T],\|x\| \leq r\right\} .
\end{aligned}
$$

Since the function $f(t, x)$ is uniformly continuous on the set $[0, T] \times\left[-r_{0}, r_{0}\right]$, the function $g(t, y)$ is uniformly continuous on the set $[0, T] \times[-N, N]$, the function $h(t, x)$ is uniformly continuous on the set $[0, T] \times\left[-r_{0}, r_{0}\right]$ and the function $u(t, s, x, y)$ is uniformly continuous on the set $[0, T] \times[0, T] \times\left[-r_{0}, r_{0}\right] \times\left[-r_{0}, r_{0}\right]$, where

$$
N=\sup \left\{\int_{0}^{t_{1}} \frac{\left|u\left(t_{1}, s, x(s), x(\lambda s)\right)\right|}{\left(t_{1}-s\right)^{1-\alpha}} d s: t_{1} \in[0, T],\|x\| \leq r_{0}\right\},
$$

(Obviously, $N<\infty$ because $u(t, s, x, y)$ is bounded on $[0, T] \times[0, T] \times\left[-r_{0}, r_{0}\right] \times$ $\left[-r_{0}, r_{0}\right]$ and $\left.\int_{0}^{t_{1}} \frac{1}{\left(t_{1}-s\right)^{1-\alpha}} d s \leq \frac{T^{\alpha}}{\alpha}\right)$, we have

$\sup \left\{\left|g\left(t_{2}, y\right)-g\left(t_{1}, y\right)\right|: t_{1}, t_{2} \in[0, T],\left|t_{2}-t_{1}\right| \leq \varepsilon,|y| \leq N\right\} \rightarrow 0$ as $\varepsilon \rightarrow 0$.

Therefore, from the last estimate we derive the following one

$$
\omega_{0}^{T}(\mathcal{F} X) \leq k \omega_{0}^{T}(X) .
$$

Hence we have

$$
\omega_{0}^{\infty}(\mathcal{F} X) \leq k \omega_{0}^{\infty}(X)
$$


From (9) and (10) and the definition of the measure of noncompactness $\mu$ given by formula (3), we obtain

$$
\mu(\mathcal{F} X) \leq k \mu(X)
$$

In the sequel let us put $B_{r_{0}}^{1}=\operatorname{Conv} \mathcal{F}\left(B_{r_{0}}\right), B_{r_{0}}^{2}=\operatorname{Conv} \mathcal{F}\left(B_{r_{0}}^{1}\right)$ and so on. In this way we have constructed a decreasing sequence of nonempty, bounded, closed and convex subsets $\left(B_{r_{0}}^{n}\right)$ of $B_{r_{0}}$ such that $\mathcal{F}\left(B_{r_{0}}^{n}\right) \subset B_{r_{0}}^{n}$ for $n=1,2, \ldots$. Since the above reasons leading to (11) holds for any subset $\mathrm{X}$ of $B_{r_{0}}$ we have

$$
\mu\left(B_{r_{0}}^{n}\right) \leq k^{n} \mu\left(B_{r_{0}}\right) \text { for any } n=1,2,3, \ldots
$$

This implies that $\lim _{n \rightarrow \infty} \mu\left(B_{r_{0}}^{n}\right)=0$. Hence, taking into account Definition 2.2 we infer that the set $Y=\bigcap_{n=1}^{\infty} B_{r_{0}}^{n}$ is nonempty, bounded, closed and convex subset of $B_{r_{0}}$. Moreover, $Y \in \operatorname{ker} \mu$. Also, the operator $\mathcal{F}$ maps $Y$ into itself.

We will prove that the operator $\mathcal{F}$ is continuous on the set $Y$. In order to do this let us fix a number $\varepsilon>0$ and take arbitrary functions $x, y \in Y$ such that $\|x-y\| \leq \varepsilon$. Keeping in mind the facts that $Y \in \operatorname{ker} \mu$ and the structure of sets belong to $\operatorname{ker} \mu$ we can find a number $T>0$ such that for each $z \in Y$ and $t \geq T$ we have that $|z(t)| \leq \varepsilon$. Since $\mathcal{F}$ maps $Y$ into itself we have that $\mathcal{F} x, \mathcal{F} y \in Y$. Thus, for $t \geq T$ we get

$$
|(\mathcal{F} x)(t)-(\mathcal{F} y)(t)| \leq|(\mathcal{F} x)(t)|+|(\mathcal{F} y)(t)| \leq 2 \varepsilon
$$

On the other hand, let us assume $t \in[0, T]$. Then we obtain

$$
\begin{aligned}
& |(\mathcal{F} x)(t)-(\mathcal{F} y)(t)| \\
\leq & l(t)|x(t)-y(t)|+\frac{m(t) n(t)|x(t)-y(t)|}{\Gamma(\alpha)} \int_{0}^{t} \frac{q(t) \Phi(|x(s)|,|x(\lambda s)|)+u^{*}(t)}{(t-s)^{1-\alpha}} d s \\
& +\frac{m(t)[n(t)|y(t)|+|h(t, 0)|]}{\Gamma(\alpha)} \int_{0}^{t} \frac{q(t) \Phi(|x(s)-y(s)|,|x(\lambda s)-y(\lambda s)|)}{(t-s)^{1-\alpha}} d s \\
\leq & \varepsilon l(t)+\frac{\left[m(t) n(t) q(t) \Phi\left(r_{0}, r_{0}\right)+m(t) n(t) u^{*}(t)\right] \varepsilon}{\Gamma(\alpha)} \int_{0}^{t} \frac{d s}{(t-s)^{1-\alpha}} \\
& +\frac{\left[m(t) n(t) q(t) r_{0}+m(t) q(t)|h(t, 0)|\right] \Phi(\varepsilon, \varepsilon)}{\Gamma(\alpha)} \int_{0}^{t} \frac{d s}{(t-s)^{1-\alpha}} \\
\leq & {\left[l(t)+\frac{\phi(t) \Phi\left(r_{0}, r_{0}\right)+\psi(t)}{\Gamma(\alpha+1)}\right] \varepsilon+\frac{\phi(t) r_{0}+\xi(t)}{\Gamma(\alpha+1)} \Phi(\varepsilon, \varepsilon) } \\
\leq & {\left[l^{*}+\frac{\phi^{*} \Phi\left(r_{0}, r_{0}\right)+\psi^{*}}{\Gamma(\alpha+1)}\right] \varepsilon+\frac{\phi^{*} r_{0}+\xi^{*}}{\Gamma(\alpha+1)} \Phi(\varepsilon, \varepsilon) . }
\end{aligned}
$$

Now, taking into account $(12),(13)$ and hypothesis $\left(a_{4}\right)$ we conclude that the operator $\mathcal{F}$ is continuous on the set $Y$.

Finally, linking all the above obtained facts about the set $Y$ and the operator 
$\mathcal{F}: Y \rightarrow Y$ and using the classical Schauder fixed point principle we deduce that the operator $\mathcal{F}$ has at least one fixed point $x$ in the set $Y$. Obviously the function $x=x(t)$ is a solution of the quadratic integral equation (1). Moreover, since $B_{r_{0}} \subset \operatorname{ker} \mu$ we have that all solutions of Eq.(1) belonging to $B_{r_{0}}$ are asymptotically stable. This completes the proof.

\section{An example}

Consider the following integral equation of fractional order

$$
\begin{aligned}
x(t)= & \frac{1}{625 \pi} \arctan (t+x(t))+\frac{1}{1000+t^{2}} \\
& +\sin \left[\frac{1}{t^{3}+1} \cdot \frac{\arctan (x(t))}{\Gamma\left(\frac{1}{3}\right)} \int_{0}^{t} \frac{\sqrt{1+|x(s)|+|x(\lambda s)|}}{(t-s)^{\frac{2}{3}}} d s\right],
\end{aligned}
$$

where $t \in \mathbb{R}_{+}$.

Observe that the above equation is a special case of Eq.(1). Indeed, if we put $\alpha=\frac{1}{3}$ and

$$
\begin{aligned}
& f(t, x)=\frac{1}{625 \pi} \arctan (t+x) \\
& g(t, x)=\frac{1}{1000+t^{2}}+\sin \left(\frac{1}{t^{3}+1} \cdot x\right) \\
& h(t, x)=\arctan x \\
& u(t, s, x, y)=\sqrt{1+|x|+|y|} .
\end{aligned}
$$

In fact we have that the function $f(t, x)=\frac{1}{2 \pi} \arctan (t+x)$ satisfies assumption $\left(a_{1}\right)$ with $l(t)=\frac{1}{625 \pi}$ and $|f(t, 0)|=f(t, 0)=\frac{1}{625 \pi} \arctan t$, being $f^{*}=0.0004$. Also, the function $g(t, x)=\frac{1}{1000+t^{2}}+\sin \left(\frac{1}{t^{3}+1} \cdot x\right)$ satisfies assumption $\left(a_{2}\right)$ with $m(t)=\frac{1}{t^{3}+1}$ and $|g(t, 0)|=g(t, 0)=\frac{1}{1000+t^{2}}$, being $g^{*}=0.001$. Next, let us notice that the function $h(t, x)=\arctan x$ satisfies assumption $\left(a_{2}\right)$ with $n(t)=1$. The function $u(t, s, x, y)=\sqrt{1+|x|+|y|}$ satisfies assumption $\left(a_{3}\right)$ with $q(t)=1, \Phi(r, r)=r$ and $u(t, s, 0,0)=1$, being $u^{*}=1$.

To check that assumption $\left(a_{4}\right)$ is satisfied let us observe that the functions $\phi, \psi, \xi, \eta$ take the form

$$
\phi(t)=\frac{t^{\frac{1}{3}}}{t^{3}+1}, \psi(t)=\frac{t^{\frac{1}{3}}}{t^{3}+1}, \xi(t)=0, \eta(t)=0 .
$$

It is easily seen that

$$
\lim _{t \rightarrow \infty} \phi(t)=\lim _{t \rightarrow \infty} \psi(t)=0 .
$$

Moreover, $\phi^{*}=\psi^{*} \cong 0.705$ and, as $\Gamma\left(\frac{4}{3}\right) \cong 0.893$. In this case inequality (4) has the form

$$
(0.0005 r+0.0004+0.001) \Gamma\left(\frac{4}{3}\right)+\left[0.705 r^{2}+0.705 r\right] \leq r \Gamma\left(\frac{4}{3}\right) .
$$

We can easily check that the above inequality is has a solution $r_{0} \in(0.007,0.259)$. Moreover, $l^{*} \Gamma\left(\frac{4}{3}\right)+\phi^{*} \Phi\left(r_{0}, r_{0}\right)+\psi^{*}<\Gamma\left(\frac{4}{3}\right)$ for all $r_{0} \in(0.007,0.259)$. Indeed, 
take $r_{0}=0.258$ we have

$$
\begin{aligned}
l^{*} \Gamma\left(\frac{4}{3}\right)+\phi^{*} \Phi\left(r_{0}, r_{0}\right)+\psi^{*} & =0.0004465+0.18189+0.705 \\
& \cong 0.887 \\
& \leq 0.893 \cong \Gamma\left(\frac{4}{3}\right) .
\end{aligned}
$$

Therefore, Theorem 3.1 guarantees that the equation (14) has a solution $x=$ $x(t)$ in the space $R_{+}$belonging to the ball $B_{r_{0}}$ with $r_{0} \in(0.007,0.259)$ and the solutions in $B_{r_{0}}$ are asymptotically stable.

Acknowledgement. This work was completed when the first author was visiting the Mathematics Section, Abdus Salam International Centre for Theoretical Physics (ICTP), Trieste, Italy as a Visiting Scientist. It is a pleasure for him to express gratitude for its financial support and the warm hospitality.

\section{References}

[1] G. Anichini and G. Conti, Existence of solutions of some quadratic integral equations, Opuscula Math. 28 (2008), no. 4, 433-440.

[2] J. Appell and P. P. Zabrejko, Nonlinear Superposition Operators, Cambridge Tracts in Mathematics 95, Cambridge University Press, 1990.

[3] I. K. Argyros, Quadratic equations and applications to Chandrasekhar's and related equations, Bull. Aust. Math. Soc. 32 (1985), no. 2, 275-292.

[4] - On a class of quadratic integral equations with perturbation, Funct. Approx. Comment. Math. 20 (1992), 51-63.

[5] J. Banaś, Measures of noncompactness in the space of continuous tempered functions, Demonstratio Math. 14 (1981), no. 1, 127-133.

[6] J. Banaś, J. Caballero, J. Rocha, and K. Sadarangani, Monotonic solutions of a class of quadratic integral equations of Volterra type, Comput. Math. Appl. 49 (2005), no. 5-6, 943-952.

[7] J. Banaś and L. Olszowy, Measures of noncompactness related to monotonicity, Comment. Math. (Prace Mat.) 41 (2001), 13-23.

[8] J. Banaś and D. O'Regan, On existence and local attractivity of solutions of a quadratic Volterra integral equation of fractional order, J. Math. Anal. Appl. 345 (2008), no. 1, $573-582$.

[9] V. C. Boffi and G. Spiga, An equation of Hammerstein type arising in particle transport theory, J. Math. Phys. 24 (1983), no. 6, 1625-1629.

[10] _ Nonlinear removal effects in time-dependent particle transport theory, Z. Angew. Math. Phys. 34 (1983), no. 3, 347-357.

[11] L. W. Busbridge, The Mathematics of Radiative Transfer, Cambridge Univ. Press, Cambridge, 1960.

[12] J. Caballero, D. O'Regan, and K. Sadarangani, On solutions of an integral equation related to traffic flow on unbounded domains, Arch. Math. (Basel) 82 (2004), no. 6, $551-563$.

[13] K. M. Case and P. F. Zweifel, Linear Transport Theory, Addison-Wesley, Reading, MA 1967.

[14] S. Chandrasekhar, Radiative Transfer, Oxford University Press, London, 1950.

[15] M. A. Darwish, On quadratic integral equation of fractional orders, J. Math. Anal. Appl. 311 (2005), no. 1, 112-119.

[16] _ On existence and asymptotic behaviour of solutions of a fractional integral equation, Appl. Anal. 88 (2009), no. 2, 169-181. 
[17] M. A. Darwish and J. Henderson, Existence and asymptotic stability of solutions of a perturbed quadratic fractional integral equation, Fract. Calc. Appl. Anal. 12 (2009), no. 1, 71-86.

[18] M. A. Darwish and K. Sadarangani, On existence and asymptotic stability of solutions of a functional-integral equation of fractional order, to appear J. Convex Anal.

[19] K. Deimling, Nonlinear Functional Analysis, Springer-Verlag, Berlin, 1985.

[20] R. Hilfer, Applications of Fractional Calculus in Physics, World Scientific, Singapore, 2000.

[21] S. Hu, M. Khavani, and W. Zhuang, Integral equations arising in the kinetic theory of gases, Appl. Anal. 34 (1989), no. 3-4, 261-266.

[22] C. T. Kelley, Approximation of solutions of some quadratic integral equations in transport theory, J. Integral Equations 4 (1982), no. 3, 221-237.

[23] A. A. Kilbas, H. M. Srivastava, and Juan J. Trujillo, Theory and Applications of Fractional Differential Equations, North-Holland Mathematics Studies, 204. Elsevier Science B.V., Amsterdam, 2006.

[24] L. Kurz, P. Nowosad, and B. R. Saltzberg, On the solution of a quadratic integral equation arising in signal design, J. Franklin Inst. 281 (1966), 437-454.

[25] R. W. Leggett, A new approach to the H-equation of Chandrasekhar, SIAM J. Math. Anal. 7 (1976), no. 4, 542-550.

[26] K. S. Miller and B. Ross, An Introduction to the Fractional Calculus and Differential Equations, John Wiley, New York, 1993.

[27] I. Podlubny, Fractional Differential Equations, Academic Press, San Diego, 1999.

[28] S. G. Samko, A. A. Kilbas, and O. I. Marichev, Fractional Integrals and Derivatives: Theory and Applications, Gordon and Breach Science Publs., Amsterdam, 1993.

[29] G. Spiga, R. L. Bowden, and V. C. Boffi, On the solutions to a class of nonlinear integral equations arising in transport theory, J. Math. Phys. 25 (1984), no. 12, 3444-3450.

[30] C. A. Stuart, Existence theorems for a class of non-linear integral equations, Math. Z. 137 (1974), 49-66.

Mohamed Abdalla Darwish

Department of Mathematics

SCIENCES FACUlTy FOR GiRLS

King AbDulaziz University

Jeddah, Saudia Arabia

AND

Department of Mathematics

FACULTY OF SCIENCE

DAMANHOUR UNIVERSITY

EGYPT

E-mail address: mdarwish@ictp.it; darwishma@yahoo.com

JOHNNY HENDERSON

Department of Mathematics

BAYLOR UNIVERSITY

WACO, TX 76798-7328, USA

E-mail address: Johnny_Henderson@baylor .edu

DONAL O'REGAN

Department of Mathematics

NATIONAL UNIVERSity OF IRELAND

Galway, Ireland

E-mail address: donal.oregan@nuigalway.ie 